\title{
Retinopatía del prematuro: Actualización en detección y tratamiento
}

\author{
Retinopathy of the premature: Update in screening and treatment
}

\author{
Aldo Bancalari M. ${ }^{\mathrm{a}}$, Ricardo Schade ${ }^{\mathrm{b}}$ \\ aDepartamento de Pediatría, Facultad de Medicina. Universidad de Concepción. Servicio de Neonatología. Hospital Guillermo Grant Benavente, \\ Concepción, Chile \\ bServicio de Oftalmología Hospital Guillermo Grant Benavente, Concepción, Chile
}

Recibido: 29 de enero de 2019; Aceptado: 14 de agosto de 2019

\section{¿Qué se sabe del tema que trata este estudio?}

La retinopatía del prematuro (RDP) es la principal causa de ceguera en la infancia en los países en vías de desarrollo. En la RDP umbral el tratamiento estándar es la fotocoagulación con láser. En las etapas pre-umbrales de la RDP, no existe actualmente ningún tipo de tratamiento establecido.

\begin{abstract}
¿Qué aporta este estudio a lo ya conocido?
En este articulo se define la población de riesgo de recién nacidos prematuros para efectuar la pesquisa de RDP y determina el momento y la frecuencia de los exámenes oftalmológicos. Se revisa el uso de agentes anti-VEGF en algunos tipos de RDP (zona I y cualquier etapa con enfermedad plus) y el uso de bloqueadores beta adrenérgicos (propranolol) en etapas pre-umbrales de la retinopatía, donde parece ser efectivo en prevenir la progresión de esta enfermedad a etapas severas
\end{abstract}

\section{Resumen}

La Retinopatía del Prematuro (RDP) es una alteración proliferativa de los vasos sanguíneos de la retina inmadura, que afecta principalmente a los recién nacidos de muy bajo peso (RNMBP) y de menor edad gestacional. El objetivo de esta revisión es describir a qué niño se debe efectuar la detección de esta enfermedad y analizar los recientes avances en su tratamiento. La detección de RDP está dirigida principalmente a los RNMBP y a $\leq$ de 32 semanas de edad gestacional, pero también se ha propuesto un criterio según edad postmenstrual. Además de la fotocoagulación con láser, tratamiento estándar en la actualidad, se han desarrollado nuevas terapias, como los agentes anti factor de crecimiento vascular endotelial (VEGF), que se han utilizado exitosamente en la retinopatía umbral, especialmente localizada en zona I, con menos efectos adversos y mejores resultados oculares a futuro. que la fotocoagulación con láser. En los últimos años, se han realizado ensayos clínicos con propranolol oral como tratamiento de la RDP, principalmente en la etapa pre-umbral (etapa 2 o 3 en zona II ó III). Este bloqueador beta-adrenérgico puede prevenir la progresión de la retinopatía en RNMBP de etapa preumbral a umbral y/o evitar la necesidad de terapias invasivas, como la fotocoagulación con láser o la administración intravítrea de agentes anti-VEGF. La fotocoagulación con láser continúa siendo el tratamiento de elección en la RDP. Los agentes anti-VEGF y el propranolol oral, evitarían la progresión de esta patología de etapa pre-umbral a umbral, y podrían complementar el tratamiento de la RDP.
Palabras clave: Retinopatía del prematuro; detección; tratamiento, bevacizumab; beta bloqueadores; propranolol 


\begin{abstract}
Retinopathy of Prematurity (ROP) is a proliferative disorder of the blood vessels of the immature retina, which affects mainly very-low-birth-weight infants (VLBW). The objective of this review is to describe to which infant the screening examination of this disease should be performed and to analyze the recent advances in the treatment of this disease, which have emerged in the last decade. The detection of this disease is mainly focused on VLBW infants and newborns $\leq 32$ weeks of gestational age. In addition to laser photocoagulation, standard treatment today, new therapies have appeared, such as the anti-VEGF agents, which have been successfully used in the threshold ROP, especially located in zone I. This therapy is less harmful than laser photocoagulation and with better ocular results in the future. In recent years, oral propranolol has been used as a treatment for ROP in clinical trials, mainly in the pre-threshold stage (stage 2 or 3 in zone II or III). This drug is a beta-adrenergic blocker that can prevent the progression of retinopathy in pre-threshold to threshold stage and/or avoid the need for invasive therapies, such as laser photocoagulation or intravitreal administration of anti-VEGF agents. Laser photocoagulation continues to be the standard treatment for ROP. New treatments have emerged for ROP, such as anti-VEGF agents and oral propranolol, which could prevent the progression of this disease from the pre-threshold to the threshold stage.
\end{abstract}

Keywords: Retinopathy of prematurity; screening; treatment; bevacizumab; beta-blockers propranolol

\section{Introducción}

La Retinopatía del Prematuro (RDP) es una alteración del desarrollo de la vascularización de la retina inmadura del Recién Nacido (RN) prematuro, principalmente de muy bajo peso (RNMBP) de nacimiento y menor edad gestacional ${ }^{1-3}$. La RDP es la primera causa de ceguera infantil en países en vías de desarrollo, como India, China y en países de Latinoamérica y de Europa del Este ${ }^{2,4}$.

\section{Clasificación de la RDP}

La Clasificación Internacional de la Retinopatía del Prematuro (ICROP $)^{5}$ establece la severidad en 5 etapas y 3 zonas, según la localización de éstas ${ }^{5}$. Ambas pueden presentar la denominada forma "plus" de la enfermedad que significa una dilatación y tortuosidad de los vasos retinianos (Tablas 1 y 2$)^{5}$.

\section{Pesquisa o detección de la RDP}

El objetivo de la detección es identificar los RN con retinopatía que requieren tratamiento, con la finalidad de evitar un déficit visual severo o ceguera. A fines de la década de los ' 80 la detección de la RDP era infrecuente en las unidades de cuidados intensivos neonatales (UCI). Sin embargo, luego de determinarse que la primera causa de ceguera en niños era la RDP, la detección de esta patología se generalizó en EE.UU., Canadá y Europa ${ }^{6,7}$. Posteriormente, con la creación de la UCIs Neonatales a fines de los ' 80 y principios de los '90 en la mayoría de los países de Sudamérica, la mayor sobrevida de los RNMBP aumentó significativamente esta patología ${ }^{7,8}$. Por lo tanto, la detección de la RDP se transformó en un problema de salud pública, siendo necesario y fundamental, formular guías clínicas de detección ${ }^{6,9-11}$.

Actualmente, la mayoría de los países han diseñado programas de tamizaje de la RDP a través de guías clínicas para su detección, considerando dos principios fundamentales: a qué neonatos evaluar y cuando iniciar la detección.

Generalmente las guías clínicas de detección de la RDP evalúan a todos los RN menores o igual a 32 semanas de edad gestacional (EG) y/o $\leq 1.500$ gramos al nacer (tabla 3$)^{6}$. Además, sugieren incluir a neonatos entre 1.500 y 2.000 gramos, que han tenido un curso clínico inestable y con diversas complicaciones, junto con requerimiento de oxígeno prolongado y factores de riesgo relacionados en la etiopatogenia de la RDP tales como: hiperoxia, hipoxia, asfixia, ventilación mecánica invasiva prolongada, apnea, hemorragia intracraneana, sepsis tardía 8,9 .

La detección debe efectuarse después de que esté dilatada la pupila, a través de oftalmoscopía binocular indirecta, con lente de 20 a 28 dioptrías, realizada por oftalmólogo con experiencia ${ }^{10,11}$. En centros asistenciales que no cuentan con este recurso humano puede realizarse con telemedicina, que implica el envío de la imagen del fondo de ojo del prematuro evaluado, a un oftalmólogo (retinólogo) con experiencia ${ }^{6}$.

\section{¿Cuándo iniciar la detección de RDP?}

El inicio de la detección de esta patología está basado en el conocimiento de la historia natural de esta 
Tabla 1. Las 5 etapas de la retinopatía del prematuro

\begin{tabular}{ll}
\hline Etapa 1 & $\begin{array}{l}\text { Delgada línea demarcatoria entre retina vascular } \\
\text { y avascular }\end{array}$ \\
Etapa 2 & $\begin{array}{l}\text { Lomo o caballete en la unión de retina vascular } \\
\text { y avascular }\end{array}$ \\
Etapa 3 & $\begin{array}{l}\text { Lomo con proliferación fibrovascular extrarre- } \\
\text { tinal }\end{array}$ \\
Etapa 4 & $\begin{array}{l}\text { Desprendimiento parcial de la retina: } \\
\text { - extra-foveal (etapa 4A) } \\
\text { - foveal (etapa 4B) }\end{array}$ \\
Etapa 5 & Desprendimiento total de la retina \\
\hline
\end{tabular}

Tabla 3. Criterios de detección o pesquisa de RDP en diferentes países ${ }^{6}$

\begin{tabular}{lc}
\hline País & $\begin{array}{l}\text { Edad Gestacional en semanas y/o } \\
\text { peso nacimiento }(\mathbf{g})\end{array}$ \\
\hline EE. UU. & $<31$ sem o $<1.500 \mathrm{~g}$ \\
Canadá & $<31 \mathrm{sem} 0<1.250 \mathrm{~g}$ \\
Reino Unido & $<31 \mathrm{sem} 0<1.250 \mathrm{~g}$ \\
Argentina & $\leq 32 \mathrm{sem} y / 01.500 \mathrm{~g}$ \\
Brasil & $\leq 32 \mathrm{sem} y / 01.500 \mathrm{~g}$ \\
Chile & $\leq 32 \mathrm{sem} y / 01.500 \mathrm{~g}$ \\
India & $<34$ sem $y / 0<1.750 \mathrm{~g}$ \\
\hline
\end{tabular}

enfermedad. La mayoría de las guías clínicas tendientes a la detección o tamizaje de la retinopatía del prematuro, indican iniciar la detección a las 4 o 6 semanas postnatal ${ }^{6,11}$. Sin embargo, en recién nacidos con EG muy inmadura menor o igual a 24 semanas, ese periodo de tiempo sería un examen muy precoz y por el contrario en recién nacidos con mayor EG, como 32 semanas podría ser tardío.

El enfoque propuesto por Reynolds y cols. ${ }^{12}$ toma en cuenta la EG del recién nacido para iniciar la detección (tabla 4); Según su estudio ${ }^{12}$, el 99\% de las retinopatías severas ocurren después de las 31 semanas de edad postmenstrual o posterior a las 4 semanas postnatales. Por lo cual el primer examen ocular se debería efectuar a las 31 semanas de edad postmenstrual en neonatos con EG menor a 27 semanas y en los mayores a 27 semanas a las 4 semanas posterior al nacimiento.

Las guías recientemente formuladas por la Academia Americana de Pediatría y la Academia Americana de Oftalmología ${ }^{11}$ también hacen mención en el inicio de la detección basado en la edad postmenstrual del $\mathrm{RN}$, debido a que el inicio de una retinopatía severa se correlaciona mejor con la edad postmenstrual, la cual comprende la EG más la edad cronológica ${ }^{11}$.
Tabla 2. Clasificación de la retinopatía del prematuro según la localización

Zona I: Área o circulo que rodea simétricamente el nervio óptico, que se extiende desde el nervio óptico, hasta 2 veces la distancia entre el nervio óptico y la fóvea. Es la zona más posterior y es la primera que se desarrolla

Zona II: Área que se extiende desde el borde de la zona I hasta la ora serrata en el lado nasal y hasta el ecuador del lado temporal

Zona III: Área en forma de media luna creciente más anterior que la zona II

Tabla 4. Edad postnatal de pesquisa de la RDP según edad gestacional*

\begin{tabular}{ccc}
\hline $\begin{array}{c}\text { Edad gestacional } \\
(\text { sem })\end{array}$ & $\begin{array}{c}\text { Edad postnatal } \\
(\text { sem })\end{array}$ & $\begin{array}{c}\text { Edad postmentrual } \\
(\text { sem })\end{array}$ \\
\hline 22 & 8 & 30 \\
23 & 7 & 30 \\
24 & 6 & 30 \\
25 & 5 & 30 \\
26 & 5 & 30 \\
27 & 4 & 31 \\
28 & 4 & 32 \\
29 & 4 & 33 \\
30 & 4 & 34 \\
31 & 4 & 35 \\
32 & 3 & 35 \\
33 & 2 & 35 \\
34 & 2 & 36 \\
\hline * pesquisa de RDP según Reynolds &
\end{tabular}

\section{Frecuencia del examen oftalmológico}

La frecuencia del examen oftalmológico depende de la etapa o la zona de la RDP, considerando siempre en optimizar el número y la frecuencia de los exámenes oculares realizado por el oftalmólogo ${ }^{6,11}$.

En pacientes con vascularización inmadura o en etapa 1 o 2 en zonas II o III de la RDP, el examen se puede efectuar cada 2 semanas. Sin embargo, cuando la vascularización es inmadura en zona I o en etapas más avanzadas, por ejemplo, etapa 2 o 3 , el examen debe ser semanal o incluso dos veces a la semana ${ }^{11}$.

En etapa 1 o 2 de la retinopatía en zona III, que ha sido tratada con láser y/o agente anti factor de crecimiento vascular endotelial (VEGF) o en etapa de franca regresión, es decir, ubicada la retinopatía en zona III, el examen oftalmológico puede ser efectuado cada 
2 o 3 semanas $^{11}$. En los casos evaluados cuyo examen ocular es normal, la detección también debe efectuarse cada dos semanas.

\section{¿Cuándo finalizar o suspender los exámenes oftal- mológicos?}

El examen ocular se detiene al lograr el paciente una vascularización completa, es decir, cuando la vascularización retiniana alcanza la ora serrata en $360^{\circ 11}$. En ausencia de enfermedad pre-umbral el examen se puede suspender a una edad postmenstrual de 50 semanas y a las 45 semanas en retinopatía no tipo $1^{11}$. Se recomienda suspender el examen oftalmológico periódico a las 65 semanas de edad postmenstrual, si se ha utilizado tratamiento con agentes anti-VEGF, porque esta terapia altera la historia natural de esta enfermedad $^{11}$. El seguimiento de los niños tratados con agentes anti-VEGF, debe continuarse, hasta que finaliza la maduración retinal, que normalmente ocurre entre los 8 a 9 años. Cuando la vascularización de la retina ha alcanzado la zona III; se recomienda suspender el examen ocular, puesto que el riesgo de un resultado adverso es muy improbable ${ }^{11}$.

\section{Tratamiento de la Retinopatía del Prematuro}

Hace 30 años, la crioterapia, fue el primer tratamiento que resultó ser efectivo para la retinopatía umbral o severa ${ }^{13}$. La crioterapia consiste en la quemadura por frío del espesor total de la retina avascular inmadura. Posteriormente, algunos investigadores observaron que el tratamiento de la retinopatía umbral con fotocoagulación con láser, tenía iguales o incluso mejores resultados que la crioterapia, al presentar menor dolor e inflamación ${ }^{14}$; constituyéndose desde entonces, hasta el presente, en la terapia estándar para la retinopatía umbral. Se entiende por retinopatía en etapa umbral aquella que se encuentra en una etapa de evolución, en la que existe una probabilidad estadística de un 50\% que progrese hacia el desprendimiento de retina (ceguera) y que habitualmente se presenta en etapa 3 y en zona II o III con enfermedad plus ${ }^{10}$. Retinopatía preumbral es aquella que se encuentra en etapa mayor a 1 y que no ha desarrollado enfermedad plus ${ }^{10}$.

El estudio ETROP ${ }^{15}$ estableció las guías actuales de tratamiento más eficaces y oportunas para la RDP de tipo 1, determinando que la fotocoagulación con láser en la retinopatía tipo 1, tiene una alta tasa de éxito visual y anatómico ${ }^{15}$. Según este estudio se definió como RDP tipo 1 aquella localizada en: Zona I, cualquier etapa con enfermedad plus; Zona I, etapa 3 sin enfermedad plus (rara); Zona II, etapa 2 o 3 con enfermedad plus. RDP tipo 2: RDP en zona I, etapa 1 y 2 sin enfermedad plus y RDP en zona II, etapa 3 sin enfermedad plus. Para la RDP tipo 2 se recomienda observación continua y frecuente. Se entiende por enfermedad plus, cuando existe dilatación y tortuosidad de los vasos retinianos en al menos 2 cuadrantes del polo posterior de la retina ${ }^{10,11}$. La mayoría de los casos tratados corresponden a pacientes con enfermedad plus, siendo muy infrecuente la retinopatía en zona I y etapa 3 sin enfermedad plus ${ }^{11,15}$. En un paciente con una retinopatía tipo 1, el tratamiento con láser y/o agente anti-VEGF, debe efectuarse antes de las 72 horas de efectuado el diagnóstico, con la finalidad de reducir el riesgo de desprendimiento de retina ${ }^{11}$.

\section{Tratamiento con láser}

El tratamiento con láser evita en más de un $80 \%$ la progresión de esta enfermedad y constituye la terapia estándar para esta patología en etapa umbral ${ }^{14}$. Este tratamiento puede presentar complicaciones tales como hemorragia vítrea, hifema, catarata, aumento de los vicios de refracción y, tal vez el peor efecto colateral que es, la destrucción de la retina periférica avascular, por lo cual el niño queda con un campo visual limitado $^{14}$. Sin embargo, tiene la ventaja de no presentar efectos sistémicos ${ }^{16}$, y se recomienda realizar el examen ocular luego de 3 a 7 días, para descartar un posible tratamiento adicional con láser en áreas donde la terapia ablativa no fue completa.

El equipo láser tiene un costo considerable, el tratamiento es demoroso y requiere dominio y destreza del oftalmólogo ${ }^{15,16}$. Por este motivo se han explorado nuevas alternativas terapéuticas, con menos complicaciones o secuelas. Uno de estos nuevos tratamientos es la utilización de agentes anti-VEGF.

\section{Tratamiento con agentes anti-VEGF: Bevacizumab}

El uso de medicamentos inhibidores del VEGF ha mostrado resultados alentadores como tratamiento de la retinopatía en etapa umbral, ya sea como monote-

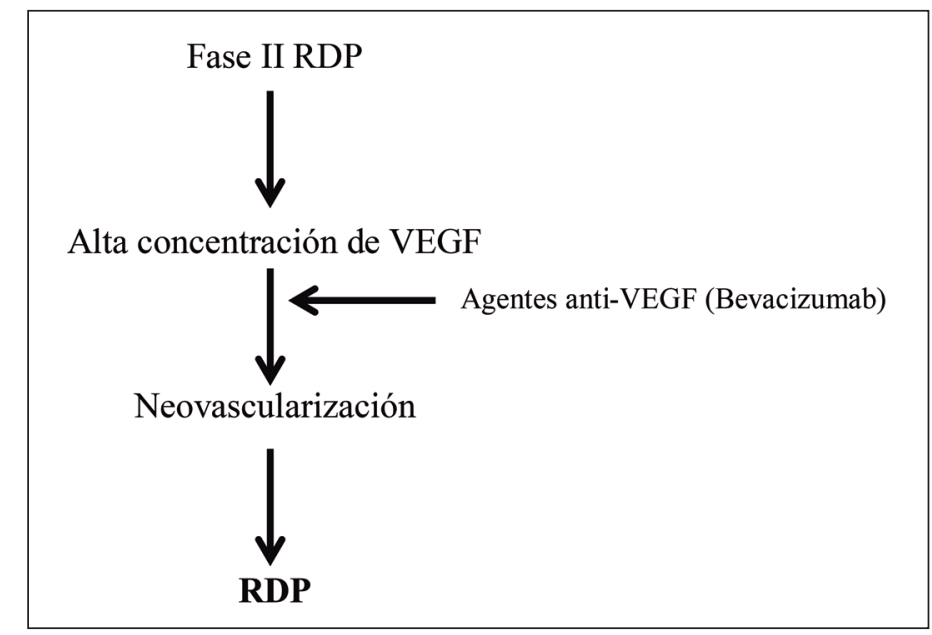

Figura 1. Mecanismos de acción del Bevacizumab. 
rapia o bien como coadyuvante a la fotocoagulación con láser ${ }^{17-20}$.

En la patogénesis de la RDP, la fase 2 se caracteriza por un aumento de los niveles circulantes de VEGF que estimula la vasoproliferación de nuevos vasos sanguíneos, que es una de las características de la retinopatía severa. Al usar un medicamento anti-VEGF se bloquea o inhibe la producción de este mediador y, por ende, se evita la formación de nuevos vasos sanguíneos (figura 1).

Existen 4 inhibidores del VEGF: el Bevacizumab (Avastin), Ranibizumab (Lucentis), Pegaptanib (Macugen) y el Aflibercept (VEGF trap) ${ }^{16}$. El más utilizado en niños prematuros y del que se dispone más experiencia es el bevacizumab ${ }^{16}$, anticuerpo monoclonal recombinante humanizado, cuyo mecanismo de acción se basa en la unión y en la consiguiente neutralización del VEGF, evitando su asociación a receptores endoteliales $^{21}$. El bevacizumab ha sido aprobado por la FDA, para tratar algunos tipos de cáncer; pero aún no ha sido aprobado para el tratamiento de la $\mathrm{RDP}^{22}$. Debido a su mecanismo de acción se ha explorado su uso en la retinopatía diabética y en la degeneración macular exudativa ${ }^{23}$

Los reportes iniciales de neonatos tratados con bevacizumab, datan del año 2007, los cuales mostraron la experiencia en un grupo reducidos de ojos, utilizándose en uno de estos reportes, la asociación con láser con respuesta favorable ${ }^{17}$. En nuestro centro se utiliza desde el año 2008, inicialmente en forma combinada con el láser y posteriormente como monoterapia ${ }^{19,20}$. Al usar bevacizumab como coadyuvante a la terapia estándar con láser, su eficacia fue de $100 \%$, al no requerirse reintervención de los ojos tratados ${ }^{19}$. En un estudio en que se utilizó bevacizumab o pegaptanib más láser en forma simultánea como tratamiento en etapa umbral, se observó una efectividad de un 90,2\% ${ }^{24}$.

En el año 2011, se publicó el primer estudio prospectivo multicéntrico aleatorizado y controlado (BEAT-ROP), con una población de 150 pacientes prematuros, que comparó la eficacia del bevacizumab versus la fotocoagulación con láser en la RDP etapa 3 plus $^{18}$. Este ensayo clínico mostró como resultado una recurrencia de un 4\% (6/140 ojos) en el grupo tratado con bevacizumab como monoterapia y un $22 \%$ (32/146 ojos) en el grupo tratados con láser. Se concluyó que el bevacizumab intravítreo comparado con el láser mostró beneficios significativos en la detención de la enfermedad en zona I, pero no en zona II posterior $^{18}$. Por el bajo número de pacientes, este estudio clínico no logró evaluar seguridad ${ }^{18}$. El seguimiento de la cohorte mostró a los dos años y medio de evolución, que los prematuros tratados con bevacizumab presentaron miopía severa solo en el 1,7\% versus el $36,4 \%$ en los tratados con láser $(\mathrm{p}<0,001)^{25}$.

Estudios realizados en Turquía, que han comparado la efectividad de los agentes anti-VEGF y del láser en el tratamiento de la RDP, han demostrado una eficacia similar entre las terapias, pero con menores alteraciones refractivas a futuro en los niños tratados con agentes anti-VEGF ${ }^{26-29}$. El meta análisis recientemente publicado que compara la eficacia de los agentes antiVEGF versus el tratamiento con láser en la retinopatía tipo 1 y umbral, concluyó que el tratamiento con láser podría ser más eficaz que la terapia con agentes antiVEGF, pero con mayores complicaciones oculares y aumento de miopía ${ }^{30}$.

El tratamiento con bevacizumab no está exento de complicaciones, tales como: hemorragia vítrea, hifema, endoftalmitis, desprendimiento de retina, catarata (1\%), recurrencia de la angiogénesis y posiblemente el efecto más crítico e importante, que es la disminución de los niveles séricos de VEGF, que es importante en el desarrollo de diversos órganos que están en formación ${ }^{31}$. Según Wu y cols. ${ }^{32}$ luego de la administración intravítrea de bevacizumab los niveles séricos de VEGF se mantienen bajos hasta por 8 semanas.

Por este motivo, aún existen varias interrogantes que deben ser aclaradas acerca del uso de los agentes anti-VEGF tales como: la dosis óptima y/o segura en

Tabla 5. Diferencias entre tratamiento con láser y Bevacizumab

\begin{tabular}{ll}
\hline Láser & Bevacizumab \\
\hline - Requiere anestesia general & - Requiere sólo sedación \\
- Requiere habitualmente intubación & - No requiere intubación \\
- Requiere experiencia del oftalmólogo & - Mínima experiencia del oftalmólogo \\
- Requiere equipo láser & - Requiere solo una jeringa (más económico) \\
- Es demoroso (1 a 2 h) & - Procedimiento rápido \\
- Destruye parte de la retina & - No destruye la retina \\
- Significativo \% de errores de refracción a futuro. & - Menor \% de errores de refracción a futuro \\
- Sin riesgo de endoftalmitis & - Riesgo muy infrecuente de endoftalmitis \\
\hline
\end{tabular}


neonatos con retinopatía umbral; tipo de retinopatía en que debe utilizarse; el momento óptimo de la administración; cuando administrar una segunda o tercera dosis ante la eventual reaparición de neovascularización; que riesgos oculares pueden ocurrir a largo plazo; o si la supresión del VEGF, por algunas semanas, podría causar disfunción de otros órganos. Algunos estudios, que han evaluado a futuro el neurodesarrollo de los niños tratados con bevacizumab han mostrado resultados contradictorios ${ }^{33-35}$, por lo cual, es importante continuar con el examen ocular hasta la edad escolar. Paralelamente es necesario efectuar mayores estudios multicéntricos aleatorizados acerca de la seguridad del bevacizumab a mediano y largo plazo. El tratamiento con láser o bevacizumab tendría indicaciones diferentes; no obstante, es importante tener presente las diferencias entre ambos tratamientos (Tabla 5).

\section{Bloqueadores Beta adrenérgicos: Propranolol}

En los últimos años, se ha utilizado el propranolol oral en el tratamiento de la retinopatía del prematuro en etapas pre-umbrales con resultados alentadores. También se ha utilizado en la prevención de esta enfermedad. En etapas pre-umbrales de la RDP, actualmente no existe ningún tipo de tratamiento, solo el control oftalmológico periódico, por lo cual disponer de una terapia que disminuya o evite la progresión a etapas más severas o umbral, podría ser beneficioso.
Según diferentes estudios, la progresión de avance de una retinopatía de etapa pre-umbral (etapa 2 y zona II ó III) a etapa umbral (etapa 3 plus), que requiere tratamiento, ocurre entre un 36 y un $49 \%{ }^{36-38}$.

El propranolol ha demostrado ser altamente efectivo en inhibir el aumento de la expresión del VEGF y la subsiguiente respuesta neovascular, que ocurre luego de la exposición a un ambiente hipóxico ${ }^{39,40}$. El posible mecanismo de acción del propranolol como tratamiento de la RDP, se esquematiza en la figura 2.

Se han publicado 5 estudios con propranolol oral como tratamiento o profilaxis de la $\mathrm{RDP}^{38,41-44}$. La primera publicación fue de Filippi y cols. ${ }^{41}$ en el año 2013, que evaluó la seguridad y efectividad en 52 recién nacidos prematuros con RDP. Dicho estudio mostró una tendencia a la reducción en la progresión de esta afección a etapa 4 y en la disminución de la necesidad de tratamiento con láser y/o bevacizumab. En el mismo año, otro estudio ${ }^{42}$ en 20 prematuros con retinopatía, observó una tendencia a favor del propranolol en reducir la necesidad de terapias invasivas, pero no en la detención de la progresión de la enfermedad. Sin embargo, en este último estudio se incluyeron pacientes con RDP en zona I y con enfermedad plus, lo que implica pacientes con mayor severidad y peor pronóstico, dificultando la interpretación de los resultados; lo que contrasta con lo reportado por Filippi y cols. ${ }^{41}$, donde la población fue mejor definida, incluyendo solo reti-

\section{Retinopatía Etapa 2 (fase 2)<smiles></smiles> \\ Hipoxia Retinal<smiles>C1C2CC12</smiles> \\ Isquemia crónica en Retina $\downarrow$ Estimulación/sobre-expresión \\ Receptores \\ B-Adrenérgicos 1 y 2

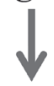 \\ Factor Hipoxia inducible (HIF)

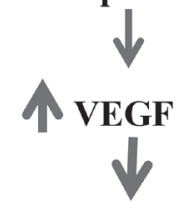 \\ Angiogenesis \\ Propranolol (bloqueo receptores)

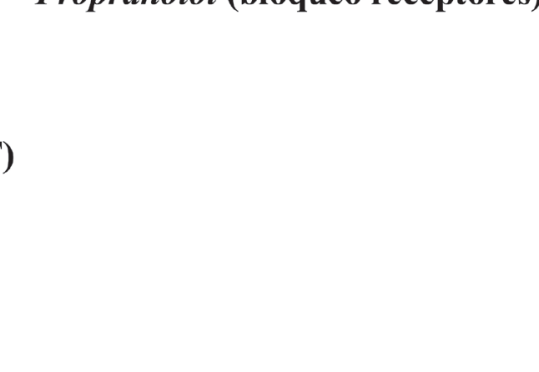

Figura 2. Posible mecanismo de acción del Propranolol (El propranolol disminuiría la sobre-expresión de factores angiogénicos: HIF, VEGF, IGF-1). 
nopatía en etapa 2 y zona II sin enfermedad plus, que corresponden a etapa pre-umbral.

En nuestro centro en el año 2012, se inició un estudio piloto con propranolol oral en RNMBP con retinopatía en etapa pre-umbral (etapa 2 o 3 , en zona II o III, sin enfermedad plus). Se evaluaron 20 prematuros con RDP, a quienes se les administró $1,5 \mathrm{mg} / \mathrm{kg} /$ día de propranolol oral, previa evaluación cardiovascular y metabólica. Posteriormente fueron comparados con un grupo control histórico, con equivalente retinopatía ${ }^{38}$. El grupo tratado con propranolol, el 90\% (18/20) no requirió ningún tipo de tratamiento, a diferencia del grupo control, en que el 52\% (14/27) no requirió ningún tratamiento, diferencia significativa $(\mathrm{p}<0,05)$. No se observaron efectos colaterales y se concluyó que este fármaco vía oral en etapas pre-umbrales de la retinopatía, puede prevenir la progresión y reducir la necesidad de terapias de rescate ${ }^{38}$.

Durante el año 2017, se publicaron dos estudios aleatorizados y controlados en RNMBP con EG menor a 32 semanas. El estudio en Turquía ${ }^{43}$ enroló 171 RNMBP, para evaluar el efecto del tratamiento con propranolol en diferentes etapas de la retinopatía y determinar su correlación con el índice de masa plaquetaria. Se observó una diferencia significativamente menor en la necesidad de láser en el grupo tratado con propranolol respecto del grupo control $(\mathrm{p}<0,02)$. Destaca que en los niños con retinopatia en etapa 1 no se encontraron diferencias en la necesidad de láser o de agentes anti-VEGF entre ambos grupos. Sin embargo, en los niños con RDP en etapa 2, el tratamiento con propranolol redujo significativamente la necesidad de terapia con láser ${ }^{43}$.

El otro estudio realizado en la India ${ }^{44}$, enroló 109 RNMBP; 55 tratados con propranolol oral en forma profiláctica y 54 controles, con el objetivo de evitar el desarrollo y la progresión de la enfermedad. El grupo con propranolol inició el tratamiento al 8vo día post natal, con $1 \mathrm{mg} / \mathrm{kg} /$ día, hasta una EG corregida de 37 semanas o hasta la vascularización completa de la retina. Se observó una tendencia a menor incidencia de retinopatía $(56,8 \%$ versus $68,6 \%)$ y una menor utilización de terapia con láser en el grupo tratado con propranolol, que no alcanzó significación estadística ${ }^{44}$. Al evaluar la incidencia de RDP en el grupo propranolol versus el grupo control se observó que la retinopatía en etapa 1 fue exactamente igual en ambos grupos $(27,4 \%)$, sugiriendo que la administración profiláctica de propranolol no sería efectiva en evitar que se desarrolle esta enfermedad ${ }^{44}$. Sin embargo, se demostró una disminución en la incidencia de retinopatías en etapas pre-umbrales (etapa 2 o etapa 3 sin enfermedad plus), tal como lo han demostrado otros estudios. En base a estos resultados se puede postular, que no se justificaría una estrategia de prevención de la RDP, con la administración profiláctica de propranolol oral en RNMBP, ya que no disminuye la incidencia de esta patología y por otro lado, expone a un porcentaje cercano al $50 \%$ de RN prematuros a un medicamento sistémico con potenciales efectos secundarios.

En nuestro centro, en base a datos propios previos ${ }^{38}$ y de la literatura ${ }^{41,42}$, iniciamos un estudio randomizado y controlado para evaluar el efecto del propranolol oral (dosis de $1,5 \mathrm{mg} / \mathrm{kg} /$ día, cada $8 \mathrm{hrs}$ ), en la progresión de las etapas pre-umbrales de la retinopatía: etapa 2 o 3 , en zona II o III, sin enfermedad plus. Se evaluaron $62 \mathrm{RN}$ menores de 32 semanas y/o $\leq \mathrm{a} 1.500 \mathrm{~g}$, de los cuales 31 recibieron propranolol oral y 31 formaron el grupo control. En el grupo con propranolol sólo 3 prematuros $(9,7 \%)$ requirieron tratamiento con láser o bevacizumab en comparación al grupo control, en que $14(45,2 \%)$ necesitaron intervención con láser o bevacizumab $(\mathrm{p}<0,01)^{45}$. Sólo un prematuro en el grupo propranolol presentó hipoglicemia en el primer día de tratamiento que no fue necesaria su suspensión $^{45}$. No se observaron otros efectos colaterales en los niños tratados con propranolol ${ }^{45}$. Se concluyó que el tratamiento con propranolol en $\mathrm{RN}$ prematuros con retinopatía pre-umbral es efectivo en prevenir la progresión de esta enfermedad y reduce la necesidad de terapias invasivas tales como el láser y/o agentes antiVEGF ${ }^{45}$.

El meta-análisis publicado el año $2018^{46}$, acerca del efecto de los $\beta$-bloqueadores en la prevención y tratamiento de la retinopatía del prematuro, demostró que el tratamiento con propranolol en los RN prematuros con retinopatía disminuye la necesidad de rescate con agentes anti-VEGF OR 0,32 [0,12, 0,86] y la necesidad de fotocoagulación con láser OR 0,54 $[0,32 ; 0,89]^{46}$.

Una revisión sistemática y metaanálisis reciente, referente al efecto del propranolol oral en la RDP, concluye que este beta-bloqueador parece ser efectivo en prevenir la progresión de esta enfermedad a etapas severas que requieren tratamiento de rescate ${ }^{47}$.

A fines del 2019 se iniciará en Europa un estudio multicéntrico, aleatorizado y controlado con un número significativo de prematuros menores de 28 semanas de EG y con un peso al nacimiento menor de 1.250 g, con retinopatía en etapa 1 o 2 con o sin enfermedad plus, con tratamiento oral de propranolol, cuyos resultados a futuro, pueden establecer la seguridad y eficacia del propranolol oral en el tratamiento de esta patología ${ }^{48}$.

\section{Cirugía}

En etapas más avanzadas de la retinopatía, como la 4 y 5 , se puede efectuar un tratamiento quirúrgico paliativo, como es la vitrectomía con pobres resultados y deterioro visual severo permanente ${ }^{49}$. 


\section{Conclusión}

En la mayoría de los países en vías de desarrollo, la detección de la RDP, está dirigida a todos los RNMBP y/o menores de 32 semanas de EG.

Actualmente los agentes anti-VEGF, se están utilizando en el tratamiento de la retinopatía umbral, especialmente en aquella localizada en zona I con enfermedad plus. Este tratamiento presenta ventajas respecto al tratamiento convencional con foto-coagulación con láser; por ser una terapia con menor pérdida de campo visual, más específica según la etiopatogenia de la enfermedad y que se puede efectuar sin anestesia general o necesidad de traslado del paciente.

Sin embargo, el tratamiento con láser continúa siendo la terapia de elección y establecida (gold stan- dard), especialmente en la retinopatía en etapa 3 plus en zona II o III.

En la actualidad dado que no existe ningún tipo de tratamiento establecido en etapas pre-umbrales de la retinopatía, disponer de una terapia como el propranolol, que evite la progresión de esta patología, es de vital importancia.

Se requieren nuevos estudios para determinar las indicaciones precisas, la seguridad y la dosis óptima, para la administración de los agentes anti-VEGF y del propranolol oral.

\section{Conflicto de intereses}

Los autores declaran no tener conflictos de interés.

\section{Referencias}

1. Jordan CO. Retinopathy of Prematurity. Pediatr Clin N Am. 2014; 61:567-77.

2. Zin A, Gole G. Retinopathy of prematurity. Incidence today. Clin Perinatol. 2013;40:185-200.

3. Bancalari A.: Retinopatía del Prematuro. En: Rizzardini M. ed: Pediatría, Santiago. Publicaciones Técnicas Mediterráneo Ltda. 1999; 260-3.

4. Kong L, Fry M, Al-Samarraie M, Gilbert C, Steinkuller PG. An update on progress and the changing epidemiology of causes of childhood blindness worldwide. J AAPOS. 2012;16:501-7.

5. International Committee for the Classification of Retinopathy of Prematurity. The International classification of retinopathy of prematurity revisited. Arch Ophthalmol. 2005; 123: 991-9.

6. Wilson C, Ells A, Fielder A. The Challenge of Screening for Retinopathy of Prematurity. Clin Perinatol. 2013;40:24159.

7. Gilbert C. Retinopathy of prematurity: a global perspective of the epidemics: population of babies at risk and implications for control. Early Hum Dev. 2008; 84:77-82.

8. Bancalari A, González R, Vásquez C, Pradenas I: Retinopathy of prematurity: incidence and associated factors. Rev Chil Pediatr. 2000;71:114-21.

9. Kim SJ, Port A, Swan R, Campbell JP, Chan RVP, Chiang MF. Retinopathy of prematurity: a review of risk factors and their clinical significance. Surv Ophthalmol. 2018;63: 618-37.

10. Gobierno de Chile. Ministerio de Salud. Guia clínica: Retinopatia del Prematuro. 2010. Disponible en: https://www.minsal. cl/portal/url/item/7222754637ab8646e04 001011f014e64.pdf (Acceso en diciembre de 2018).

11. Fierson WM. Screening Examination of Premature Infants for Retinopathy of Prematurity. Pediatrics. 2018;142:1-9.

12. Reynolds JD, Dobson V, Quinn GE, et al. CRYO-ROP and LIGHT-ROP Cooperative Groups. Evidence-based screening criteria for retinopathy of prematurity: natural history data from CRYO-ROP and LIGHT-ROP Studies. Arch Ophthalmol. 2002;120:1470-6.

13. Cryotherapy for Retinophaty of Prematurity Cooperative Group. Multicentre trial of cryotherapy for retinophaty of prematurity: preliminary results. Arch Ophthalmol. 1988; 106:4719.

14. The Laser ROP Study Group. Laser therapy for retinopathy of prematurity. Arch Ophthalmol. 1994;112:154-6.

15. Early Treatment for Retinopathy of Prematurity Cooperative Group (ETROP). Revised Indications for the treatment of retinopathy of prematurity: results of the early treatment for retinopathy of prematurity randomized trial. Arch Ophthalmol. 2003;121:168494.

16. Wallace D, Wu K. Current and Future Trends in Treatment of Severe Retinopathy of Prematurity. Clin Perinatol. 2013;40:297-310.

17. Law JC, Recchia FM, Morrison D, Estes R. Intravitreal bevacizumab (Avastin) as adjunctive treatment for retinopathy of prematurity (ROP). JAAPOS 2010;14:610.

18. Mintz-Hittner HA, Kennedy KA, Chuang AZ. Efficacy of intravitreal bevacizumab for stage 3+ retinopathy of prematurity. N. Engl J Med 2011;364:603-15.
19. Bancalari A, Schade R, Peña R, Pavez N. Tratamiento de la retinopatía del prematuro con bevacizumab intravítreo. Rev Chil Pediatr. 2013;84:300-7.

20. Bancalari A, Schade R, Peña R, Pavez N. Intravitreal bevacizumab as single drug therapy for retinopathy of prematurity in 12 patients. Arch Argent Pediatr. 2014;112:160-3.

21. Shih T, Lindley C. Bevacizumab: an angiogénesis inhibitor for the treatment of solid malignancies. Clinical Therapeutics 2006;28:1779-802.

22. FDA. Avastin Approval History. U.S. Food and Drug Administration. Disponible en http://www. accessdata.fda.gov/drugsatfda_docs/ label/2009/125085s01691bl.pdf (Acceso en octubre de 2012).

23. Avery RL, Pearlman J. Pieramici DJ, et al. Intravitreal bevacizumab (Avastin) in the treatment of proliferative diabetic retinopathy. Ophthalmology. 2006;113:1695-705.

24. Autrata R, Senková K, Holousová M, Dolezel Z, Krejcírová I, Borek I. Effects of intravitreal pegaptanib or bevacizumab and laser in treatment of threshold retinopathy of prematurity in zone I and posterior zone II-four years results. Cesk Slov Oftalmol. 2012;68:29-36.

25. Geloneck MM, Chuang AZ, Clark WL, et al. BEAT-ROP Cooperative Group. Refractive outcomes following bevacizumab monotherapy compared with conventional laser treatment a randomized clinical trial. JAMA Ophthalmol. 2014;132:1327-33.

26. Gunay M, Sukgen EA, Celik G, Kocluk Y. Comparison of Bevacizumab, Ranibizumab and Laser Photocoagulation in the Treatment of Retinopathy of Prematurity in Turkey. Curr Eye 
Research. 2017;42:462-9.

27. Sukgen EA, Kocluk Y. Comparison of clinical outcomes of intravitreal ranibizumab and aflibercept treatment for retinopathy of prematurity. Graefes Arch Clin Exp Ophthalmol. 2018;256:1-7.

28. Kabatas EU, Kurtul BE, Özer PA, Kabatas N. Comparison of Intravitreal Bevacizumab, Intravitreal Ranibizumab and Laser Photocoagulation for Treatment of Type 1 Retinopathy of Prematurity in Turkish Preterm Children. Current Eye Research. 2017;42:1054-8.

29. Gunay M, Celik G, Gunay BO, Aktas A, Karatekin G, Ovali F. Evaluation of 2 year outcomes following intravitreal bevecizumab (IVB) for aggressive posterior retinopathy of prematurity. ARQ Bras Oftalmol. 2015;78:300-4.

30. Li Z, Zhang Y, Liao Y, Zeng R, Zeng P, Lan Y. Comparison of efficacy between anti-vascular endothelial growth factor (VEGF) and laser treatment in Type-1 and threshold retinopathy of prematurity (ROP). BMC Ophthalmology 2018;18:110.

31. Jamrozy-Witkowska A, Kowalska K, Jankowska-Lech I, Terelak-Borys B, Nowosielska A, Grabska-Liberek I. Complications of intravitreal injections-own experience. Klin Oczna. 2011;113:127-31

32. Wu WC, Lien R, Liao PJ, et al. Serum levels of vascular endothelial growth factor and related factors after intravitreous bevacizumab injection for retinopathy of prematurity. JAMA Ophthalmol. 2015;133:391-7.

33. Honda S, Hirabayashi H, Tsukahara Y, Negi A. Acute contraction of the proliferative membrane after an intreavitreal injection of bevacizumab for advanced retinopathy of prematurity. Graefes Arch Clin Exp Ophthalmol. 2008;246:1061-3.

34. Morin J, Luu TM, Superstein R, et al. Neurodevelopmental outcomes following bevacizumab injections for retinopathy of prematurity. Pediatrics. 2016;137:1-8.

35. Araz-Ersan B, Kir N, Tuncer S, et al. Preliminary anatomical and neurodevelopmental outcomes of intravitreal bevacizumab as adjunctive treatment for retinopathy of prematurity. Curr Eye Res. 2015;40:585-91.

36. Austeng D, Källen KB, Hellström A, Tornqvist K, Holmström GE. Natural history of retinopathy of prematurity in infants born before 27 weeks gestation in Sweden. Arch Ophthalmol. 2010;128:1289-94.

37. Chen YH, Lien RI, Tsai S, et al. Natural history of retinopathy of prematurity: two-year outcomes of a prospective study. Retina. 2015;35:141-8.

38. Bancalari A, Schade R, Muñoz T, Lazcano C, Parada R, Peña R. Oral propranolol in early stages of retinopathy of prematurity. J. Perinat Med. 2016;44:499-503.

39. Lavine JA, Sang Y, Wang S, Ip MS, Sheibani N. Attenuation of choroidal neovascularization by $\beta(2)$ adrenoreceptor antagonism: JAMA Ophthalmol. 2013; 13:376-82.

40. Ristori C, Filippi L, Dal Monte M, et al. Role of the adrenergic system in a mouse model of oxygen-induced retinopathy: antiangiogenic effects of beta-adrenoreceptor blockade. Invest Ophthalmol Vis Sci. 2011;52:155-70.

41. Filippi L, Cavallaro G, Bagnoli P, et al. Oral propranolol for retinopathy of prematurity: risks, safety concerns, and perspectives. J Pediatr. 2013;163:1570-7.
42. Makhoul IR, Peleg O, Miller B, et al. Oral propranolol versus placebo for retinopathy of prematurity: a pilot, randomised, double-blind prospective study. Arch Dis Child. 2013;98:565-7.

43. Korkmaz L, Bastuğ O, Ozdemir A, et al. The efficacy of propranolol in retinopathy of prematurity and its correlation with the platelet mass index. Current Eye Research 2017;42:88-97

44. Sanghvi K, Kabra NS, Padhi P, Singh U, Dash SK, Avasthi BS. Prophylactic propranolol for prevention of ROP and visual outcome at 1 year (PreROP trial). Arch Dis Child Fetal Neonetal Ed. 2017;102:F389-94.

45. Bancalari A, Schade R, Lazcano C, Muñoz T, Sepúlveda G. Treatment of Retinopathy of Prematurity with Propranolol: Randomized Control Trial. E-PAS 2018, 2665.

46. Kaempfen S, Neumann RP, Jost K, Schulzke SM. Beta-blockers for prevention and treatment of retinopathy of prematurity in preterm infants (Review). Cochrane Database Syst Rev 2018;3:CD011893.

47. Stritzke A, Kabra N, Kaur S, Robertson HL, Lodha A. Oral propranolol in prevention of severe retinopathy of prematurity: a systematic review and meta-analysis. J Perinatol. 2019; 39: 158494.

48. Bürher C, Erdeve O, Bassler D, Bar-Oz B. Oral propranolol for prevention of threshold retinopathy of prematurity (ROPROP): protocol of a ramdomised controlled trial. BMJ Open 2018;8:1-7.

49. Ram Dogra M, Katoch D and Dogra M. An update of retinopathy or prematurity. Indian J Pediatr 2017;84:930-6. 